\title{
Bronchoscopy in Daily Practice: Back to Basics
}

\author{
M. Noppen \\ Interventional Endoscopy Clinic, Academic Hospital, Brussels, Belgium
}

At the turn of a century, people typically try to imagine what the future will hold. When asking a bronchoscopist, quite a spectacular scenario has been described: 'Airways carrying a suspicious lesion are entered by a detached computer-driven endoscope capsula, which is navigated according to virtual 3D bronchoscopy imaging. Fluorescence imaging, microconfocal scanning microscopy, optical coherence tomography and ultrasound confirm and localize up to a microscopical level the boundaries of the suspicious lesion. All imaging data are fed to a robot, guiding a molecular nanoknife that cuts out the lesion; local cytotoxic, immunologic or genetic agents are installed into the tumor bed. High-energy sources are inserted by the robot. Advanced computer and communication technology allow this intervention to be performed over long distances.'

This has not been written by Isaac Asimov, Arthur C. Clarke, Jules Verne or Nostradamus, but by Heinrich Becker [1], a leading authority in bronchoscopy, in a very readable perspective article on bronchoscopy in the future. Just after having read this article - and still 'high' on this sci-fi high tech bronchoscopy stuff - I was invited to write an editorial to an article by Karahalli et al. [2] appearing in this issue of Respiration, in which a simple question is asked: If you see a cancerous lesion in the airways during bronchoscopy, is it useful to take cytological specimens (brushing, washing), in addition to forceps biopsies, or not? Wham, back to earth: Is it really true that, whilst some of us are working on voice-activated, 3D computer-aided nanorobots entering malignant lesions for selective, intelligent surgery or genetic repair, others are still asking - uh, basic - questions such as: 'Should we wash a tumor if we see one?

Yes it is, and rightfully so.

It is remarkable, indeed, that many 'routine jobs' are performed the way they are, because 'we were taught so', or because 'everybody does it', or because 'it has always worked well'. It is because such 'simple' questions continue to be asked that the quality of care slowly but constantly has improved. This process, in fact, is a cornerstone in the scientific method of evidence-based medicine. Karahalli et al. [2] therefore are to be complimented on asking their 'simple' question. Did they come up with an answer? Yes and no, unfortunately.

What are the issues here?

(1) A nondiagnostic bronchoscopy should be avoided $[3,4]$ : bronchoscopy is expensive, time-consuming and uncomfortable for the patient. Adding brushing and washing to the forceps biopsies only marginally increases the length, cost or complication rate of the procedure (and if the pathologist's time or money is of concern, then after obtaining all possible specimens, they may be processed in chronological order, as needed, based on the diagnostic yield) [4]. Although the diagnostic yield in case of a visible tumor is high (around $80-85 \%$ ), and therefore difficult to improve, most authors have shown that a combination of biopsy, washing and brushing is superior to a biopsy alone, irrespective of the type of lesion [5-9]. Based on these data, there seems to be little doubt on whether cytopathological sampling should be added to biopsies.

\begin{tabular}{ll}
\hline KARGER & ( ) 2001 S. Karger AG, Basel \\
Fax +41 613061234 & 0025-7931/01/0686-0564\$17.50/0 \\
$\begin{array}{l}\text { E-Mail karger@karger.ch } \\
\text { www.karger.com }\end{array}$ & $\begin{array}{l}\text { Accessible online at: } \\
\text { www.karger.com/journals/res }\end{array}$
\end{tabular}

Marc Noppen, MD, PhD

Head, Interventional Endoscopy Clinic, Academic Hospital AZ-VUB

101, Laarbeeklaan

B-1090 Brussels (Belgium)

Tel. +32 247760 10, Fax +32 247760 19, E-Mail marc.noppen@az.vub.ac.be 
(2) The marginal increase of the diagnostic yield when adding cytology to biopsy, however, seems to vary quite importantly, from $11-12 \%[5,6]$ to $5 \%$ [10], or only $2.9 \%$ [11]. On the other hand, others have found the cytological yield to be even superior to biopsy [12,13]. The reason(s) for this disparity are unknown, but may relate to the differences in tumor types and locations in the various studies, and to individual examiner- and center-related differences in specimen handling and interpretation (number and site of biopsies, specimen processing, (cyto)pathologists' interpretation). Based on these data, the question of whether to add cytopathological sampling to biopsy is less unequivocal, and seems to depend - to a certain degree on 'local', perhaps less controllable parameters.

(3) Finally, Karahalli et al. [2] specifically compared two cytopathological sampling techniques - brushing and washing - which until now have mostly been discussed together. They found, rather surprisingly, that washing gave no additional diagnostic yield, whereas brushing increased the diagnostic yield from 82.7 to $88.8 \%$. Based on these data, the authors conclude that a routine adding of bronchial washings does not increase the yield of forceps biopsy in endoscopically visible lesions. In their cen- ter, using their overall methodology, and in their patients, they are probably right.

The question is - also taking into account other data: Can these findings be extrapolated to every bronchoscopist's practice? Probably not: We for instance routinely perform washing (and less often brushing) after having taken biopsies, and find an exclusively positive cytology in about $10 \%$.

In conclusion, notwithstanding the findings of Karahalli et al. [2], which certainly deserve to be controlled by others in their respective settings, and taking into account the body of published data on the subject, I as others [4, 9] would propose that one should continue to obtain additional cytopathological specimens (brushings and washings), since they are safe, inexpensive and minimal timeconsuming by-products of bronchoscopy, and may help to avoid repeated endoscopies in a significant number of patients. Nevertheless, whereas Karahalli et al. probably have changed their guidelines after having obtained their evidence-based data, they have invited us to think about (and maybe even reconsider) our practices, and not to keep doing things 'just because we are used to doing them in a certain way'.

\section{References}

1 Becker HD: Bronchoscopy. Year 2001 and beyond. Clin Chest Med 2001;22:225-239.

2 Karahalli E, Yilmaz A, Türker H, Özvaran K: Usefulness of various diagnostic techniques during fiberoptic bronchoscopy for endoscopically visible lung cancer: Should cytologic examinations be performed routinely? Respiration 2001;68:611-614.

3 Mehta AC: Flexible bronchoscopy in the 21st century. Clin Chest Med 1999;20:xiii.

4 Mehta AC: Wash or not to wash, brush or not to brush? That is the question. J Bronchol 2000;7:293-294.

5 Mak VHF, Johnston IDA, Hetzel MR, Grubb $\mathrm{C}$ : Value of washings and brushings at fiberoptic bronchoscopy in the diagnosis of lung cancer. Thorax 1990;45:373-376.
6 Lam WK, So SY, Hsu C, Yu DY: Fiberoptic bronchoscopy in the diagnosis of bronchial cancer: Comparison of washing, brushings and biopsies in central and peripheral tumors. Clin Oncol 1983;9:35-42.

7 Saita S, Tanzillo A, Riscica C, Potenza E, D'Arrigo M: Bronchial brushing and biopsy: A comparative evaluation in diagnosing visible bronchial lesions. Eur J Cardiothorac Surg 1990;4: 270-272.

8 Chaudhary BA, Yoneda K, Burki NK: Fiberoptic bronchoscopy: Comparison of procedures used in the diagnosis of lung cancer. $\mathrm{J}$ Thorac Cardiovasc Surg 1978;76:33-37.

9 Williams T, Bowie PES: The bronchoscopic diagnosis of lung cancer. A prospective correlation between the bronchoscopic appearances and cytology and biopsy results. J Bronchol 2000; 7:295-300.
10 Govert JA, Kopita JM, Matchar D, Kussin PS, Samuelson WM: Cost-effectiveness of collecting routine cytologic specimens during fiberoptic bronchoscopy for endoscopically visible lung tumor. Chest 1996;109:451-456.

11 Chau CH, Yew WW, Wong PC, Lee J, Wong $\mathrm{CF}$ : Usefulness of collecting routine cytologic specimens during fiberoptic bronchoscopy for endoscopically visible and non-visible lung carcinoma. Chest 1997;111:522-523.

12 Kvale PA, Bode FR, Klini S: Diagnostic accuracy in lung cancer: Comparison of techniques used in association with flexible fiberoptic bronchoscopy. Chest 1976;69:752-757.

13 Piaton E, Grillet-Ravigneaux MH, Saugier B, Pellet H: Prospective study of combined use of bronchial aspirates and biopsy specimens in diagnosis and typing of centrally located lung tumors. BMJ 1995;310:624-627. 